\title{
The global solution of anisotropic fourth-order Schrödinger equation
}

Hailing Su' and Cuihua Guo ${ }^{1 *}$

${ }^{*}$ Correspondence: gchzjq@sxu.edu.cn

'School of Mathematical Sciences, Shanxi University, Taiyuan, P.R. China

\begin{abstract}
This paper studies the global existence of solutions in Sobolev space for anisotropic fourth-order Schrödinger type equation: $i u_{t}+\Delta u+a \sum_{i=1}^{d} u_{x_{i} x_{i} x_{i} x_{i}}+b|u|^{\alpha} u=0, x \in R^{n}$, $t \in R, 1 \leq d<n$ under the initial conditions: $u(x, 0)=\varphi(x), x \in R^{n}$. By using the Banach fixed point theorem, we obtain the existence, the uniqueness, the continuous dependence and the decay estimate of the solution on the initial value in anisotropic Sobolev spaces $H_{\vec{y}}^{s_{1}, \rho} H_{\vec{z}}^{s_{2}, r}$.
\end{abstract}

MSC: 35055

Keywords: Anisotropic fourth-order Schrödinger equation; Global solution; Small initial value; Banach fixed point theorem

\section{Introduction}

In this paper we consider the initial value problem of the following anisotropic fourthorder nonlinear Schrödinger equation:

$$
\left\{\begin{array}{l}
i u_{t}+\Delta u+a \sum_{i=1}^{d} u_{x_{i} x_{i} x_{i} x_{i}}+b|u|^{\alpha} u=0, \quad x \in R^{n}, t \in R, 1 \leq d<n, \\
u(x, 0)=\varphi(x), \quad x \in R^{n}
\end{array}\right.
$$

where $a<0, \alpha>0, b$ are real numbers. $u(x, t)$ is unknown complex function, $\varphi(x)$ is the given initial value data. The above equations can be used to describe some physical phenomena. For example, [1] used (1.1) to describe the propagation of solitary waves in optical fiber arrays. [2] used (1.1) to describe the propagation of ultrashort laser pulses in a plane waveguide medium. The physical background of the equation is also given in [3-5].

For the isotropic fourth-order Schrödinger equation $(d=n)$, there are many results. For the Cauchy problem, in [6], they obtained the local well-posedness in $C\left([0, T), H^{\gamma}\left(R^{n}\right)\right)$ for some $\gamma$, also see [7-11] and the references cited therein. And they also obtained the global well-posedness in $C\left(R, H^{2}\left(R^{n}\right)\right)$. For the initial-boundary problem, in [12], Ozsari obtained the local well-posedness in $C\left([0, T), H^{s}(0,+\infty)\right)$ and the global well-posedness in $C\left(R, H^{2}(0,+\infty)\right)$; In [13], for the low regularity $s<\frac{1}{2}$, they obtained the local wellposedness in $C\left([0, T), H^{s}(0,+\infty)\right)$.

For the anisotropic fourth-order Schrödinger equation $(d<n)$, there are some conclusions about the mathematical study of such equations. We have obtained the existence of the local solution of the problem in isotropic Sobolev space $C\left([-T, T], H^{S}\left(R^{n}\right)\right)$ in [14]. The

(c) The Author(s) 2019. This article is distributed under the terms of the Creative Commons Attribution 4.0 International License (http://creativecommons.org/licenses/by/4.0/), which permits unrestricted use, distribution, and reproduction in any medium, provided you give appropriate credit to the original author(s) and the source, provide a link to the Creative Commons license, and indicate if changes were made. 
existence of global or almost global solutions for small initial value in isotropic Sobolev space $\dot{H}_{p}^{s}\left(R^{n}\right)$ has been studied in [15]. The local existence of the solution in time and space $L^{q}\left(I, L^{r}\left(R^{n}\right)\right)$ and $L^{q}\left(I, H_{a}^{1}\left(R^{n}\right)\right)\left(H_{a}^{1}\left(R^{n}\right)=\left\{u \in L^{2}\left(R^{n}\right), u_{x_{1}}, u_{x_{2}}, u_{x_{1} x_{1}} \in L^{2}\left(R^{n}\right)\right\}\right)$ is obtained by Banach fixed point theorem for the case $d=1$, furthermore, the global existence of the solution is obtained by conservation law in [16]. Some local existence results is obtained in isotropic Sobolev space $C\left(I, H^{s}\left(R^{2}\right)\right)$ on initial value problem of the anisotropic nonlinear sixth order Schrödinger equation in [17]. The asymptotic behavior in time of the solution has been obtained and it scatters to a solution of the linearized equation as $t \rightarrow \infty$ in [18]. It can be seen from the form of equation (1.1) that higher derivatives are not derived in every direction, so it is natural to think of such problems in anisotropic Sobolev spaces. In [19], the existence of local solutions of problem (1.1) in anisotropic Sobolev space $H_{\vec{y}}^{s_{1}}\left(R^{d}\right) H_{\vec{z}}^{s_{2}}\left(R^{n-d}\right)$ is given, but the global well-posedness is not discussed. In [20], we also obtain the global existence in anisotropic Sobolev space $C\left(R, W_{2}^{3, d}\left(R^{n}\right)\right)$ for the sixth order nonlinear Schrödinger equation by energy method, where $W_{2}^{3, d}\left(R^{n}\right)=\left\{u \mid u, u_{x_{j}} \in L^{2}\left(R^{n}\right), j=1, \ldots, n, u_{x_{i} x_{i}}, u_{x_{i} x_{i} x_{i}} \in L^{2}\left(R^{n}\right), i=1, \ldots, d(<n)\right\}$. But $W_{2}^{3, d}\left(R^{n}\right)$ is only an integer order Soblev space, we will study the global solution of (1.1) in anisotropic fractional order Sobolev space.

In this paper, we will give the existence, the uniqueness, the continuous dependence on the initial value and the decay estimate of the global solution for the small initial value problem (1.1) in anisotropic Sobolev spaces $H_{\vec{y}}^{s_{1}, \rho} H_{\vec{z}}^{s_{2}, r}$.

Before stating our main results, we will introduce some notations.

We take $\vec{y}=\left(x_{1}, x_{2}, \ldots, x_{d}\right), \vec{z}=\left(x_{d+1}, x_{d+2}, \ldots, x_{n}\right)$, thus $x=(\vec{y}, \vec{z})$. We denote $I_{1}\left(s_{1}\right)=\left[0, \frac{d}{2}\right)$, $I_{2}\left(s_{1}\right)=\left\{\frac{d}{2}\right\}, I_{3}\left(s_{1}\right)=\left(\frac{d}{2},[\alpha]\right] ; I_{1}\left(s_{2}\right)=\left[0, \frac{n-d}{2}\right), I_{2}\left(s_{2}\right)=\left\{\frac{n-d}{2}\right\}, I_{3}\left(s_{2}\right)=\left(\frac{n-d}{2},[\alpha]\right]$, where $[\alpha]$ represents the maximum integer which does not exceed $\alpha$. The Riesz potential $I^{\mu} \varphi=$ $F^{-1}\left(|\xi|^{\mu} \tilde{\varphi}(\xi)\right), \sim$ is a Fourier transformation, and $F^{-1}$ is the Fourier inverse transformation. We denote $\nabla_{\vec{y}}^{s_{1}} \varphi=\int_{R^{d}} e^{i\left(x_{1} \xi_{1}+x_{2} \xi_{2}+\cdots+x_{d} \xi_{d}\right)}\left|\xi_{1}^{2}+\xi_{2}^{2}+\cdots+\xi_{d}^{2}\right|^{\frac{s_{1}}{2}} \hat{\varphi}\left(\xi_{1}, \xi_{2}, \ldots, \xi_{d}, x_{d+1}\right.$, $\left.\ldots, x_{n}\right) d \xi_{1} d \xi_{2} \ldots d \xi_{d}, \nabla_{\vec{z}}^{s_{2}} \varphi=\int_{R^{n-d}} e^{i\left(x_{d+1} \xi_{d+1}+x_{d+2} \xi_{d+2}+\cdots+x_{n} \xi_{n}\right)}\left|\xi_{d+1}^{2}+\xi_{d+2}^{2}+\cdots+\xi_{n}^{2}\right|^{\frac{s_{2}}{2}} \hat{\varphi}\left(x_{1}\right.$, $\left.x_{2}, \ldots, x_{d}, \xi_{d+1}, \ldots, \xi_{n}\right) d \xi_{d+1} d \xi_{d+2} \cdots d \xi_{n}$, where $s_{1} \geq 0, s_{2} \geq 0$. The operator $S(t) g=$ $F^{-1}\left(e^{-i\left[|\xi|^{2}-a\left(\left|\xi_{1}\right|^{4}+\left|\xi_{2}\right|^{4}+\cdots+\left|\xi_{d}\right|^{4}\right)\right] t} \tilde{g}\right)$.

$L^{r}\left(R^{n}\right)$ is Banach space with the norm $\|f\|_{L^{r}\left(R^{n}\right)}=\left(\int_{R^{n}}|f(x)|^{r} d x\right)^{\frac{1}{r}} . H^{s, r}\left(R^{n}\right)$ is Banach space with the norm $\|f\|_{H^{s, r}\left(R^{n}\right)}=\|f\|_{L^{r}\left(R^{n}\right)}+\left\|I^{s} f\right\|_{L^{r}\left(R^{n}\right)}$. For simplicity, we define $L_{\vec{z}}^{r^{\prime}} \equiv L_{\vec{z}}^{r^{\prime}}\left(R^{n-d}\right), L_{\vec{y}}^{r} L_{\vec{z}}^{q} \equiv L_{\vec{y}}^{r}\left(R^{d}\right) L_{\vec{z}}^{q}\left(R^{n-d}\right)=\left\{g(\vec{y}, \vec{z}) \mid\|\| g(\vec{y}, \cdot)\left\|_{L_{\vec{z}\left(R^{n-d}\right)}^{q}}\right\|_{L_{\vec{y}\left(R^{d}\right)}^{r}}<+\infty\right\}, H_{\vec{y}}^{s_{1}, \rho} H_{\vec{z}}^{s_{2}, \gamma} \equiv$ $H_{\vec{y}}^{s_{1}, \rho}\left(R^{d}\right) H_{\vec{z}}^{s_{2}, \gamma}\left(R^{n-d}\right)=\left\{g(\vec{y}, \vec{z}) \mid\|\| g(\vec{y}, \cdot)\left\|_{H_{\vec{z}\left(R^{n-d}\right)}^{s_{2}, \gamma}}\right\|_{\left.H_{\vec{y}}^{s_{1}, \rho} R^{d}\right)}<+\infty\right\}$. Especially, $H_{\vec{y}}^{s_{1}} H_{\vec{z}}^{s_{2}} \equiv$ $H_{\vec{y}}^{s_{1}, 2}\left(R^{d}\right) H_{\vec{z}}^{s_{2}, 2}\left(R^{n-d}\right)$.

Condition 1.1 For the case $2 n-d>4$, we have the following six subcases:

$$
\left\{\begin{array}{lll}
s_{1} \in I_{1}\left(s_{1}\right), & s_{2} \in I_{1}\left(s_{2}\right), & \alpha \in\left(\frac{-\left(2 n-d-4 s_{2}-2 s_{1}-4\right)+\sqrt{\left(2 n-d-4 s_{2}-2 s_{1}-4\right)^{2}+32\left(2 n-d-4 s_{2}-2 s_{1}\right)}}{2\left(2 n-d-4 s_{2}-2 s_{1}\right)},\right. \\
& & \left.\frac{8}{2 n-d-4 s_{2}-2 s_{1}-4}\right), \\
s_{1} \in I_{1}\left(s_{1}\right), & s_{2} \in I_{2}\left(s_{2}\right), & \alpha \in\left(\frac{-\left(2 n-d-2 s_{1}-4\right)+\sqrt{\left(2 n-d-2 s_{1}-4\right)^{2}+32\left(2 n-d-2 s_{1}\right)}}{2\left(2 n-d-2 s_{1}\right)}, \frac{8}{2 n-d-2 s_{1}-4}\right), \\
s_{1} \in I_{1}\left(s_{1}\right), & s_{2} \in I_{3}\left(s_{2}\right), & \alpha \in\left(\frac{-\left(d-2 s_{1}-4\right)+\sqrt{\left(d-2 s_{1}-4\right)^{2}+32\left(d-2 s_{1}\right)}}{2\left(d-2 s_{1}\right)}, \frac{8}{d-2 s_{1}-4}\right), \\
s_{1} \in I_{2}\left(s_{1}\right), & s_{2} \in I_{1}\left(s_{2}\right), & \alpha \in\left(\frac{-\left(2 n-d-4 s_{2}-4\right)+\sqrt{\left(2 n-d-4 s_{2}-4\right)^{2}+32\left(2 n-d-4 s_{2}\right)}}{2\left(2 n-d-4 s_{2}\right)}, \frac{8}{2 n-d-4 s_{2}-4}\right), \\
s_{1} \in I_{2}\left(s_{1}\right), & s_{2} \in I_{2}\left(s_{2}\right), & \alpha \in\left(\frac{-(2 n-d-4)+\sqrt{(2 n-d-4)^{2}+32(2 n-d)}}{2(2 n-d)}, \frac{8}{2 n-d-4}\right), \\
s_{1} \in I_{2}\left(s_{1}\right), & s_{2} \in I_{3}\left(s_{2}\right), & \alpha \in\left(\frac{-(d-4)+\sqrt{(d-4)^{2}+32 d}}{2 d}, \frac{8}{d-4}\right) .
\end{array}\right.
$$


Condition 1.2 For the case $n-d>2$, we need the following condition:

$$
\left\{\begin{array}{lll}
s_{1} \in I_{3}\left(s_{1}\right), & s_{2} \in I_{2}\left(s_{2}\right), & \alpha \in\left(\frac{-(n-d-2)+\sqrt{(n-d-2)^{2}+16(n-d)}}{2(n-d)}, \frac{4}{n-d-2}\right), \\
s_{1} \in I_{3}\left(s_{1}\right), & s_{2} \in I_{3}\left(s_{2}\right), & \alpha \in\left(\frac{-\left(n-d-2 s_{2}-2\right)+\sqrt{\left(n-d-2 s_{2}-2\right)^{2}+16\left(n-d-2 s_{2}\right)}}{2\left(n-d-2 s_{2}\right)}, \frac{4}{n-d-2 s_{2}-2}\right) .
\end{array}\right.
$$

The main results are as follows.

Theorem 1.1 For the small initial value $\varphi(x)$ satisfying the condition $\|[S(t) \varphi](x)\|_{X} \leq \varepsilon$, the initial value problem has a unique global solution in the some subspaces of the space $X=$ $\left\{u:(0,+\infty) \rightarrow H_{\vec{y}}^{s_{1}, \rho} H_{\vec{z}}^{s_{2}, r} \mid\|u\|_{X}=\sup _{t>0} t^{\theta}\|u(t)\|_{H_{\bar{y}}^{s_{1}, \rho}} H_{\vec{z}}^{s_{2}, r}<+\infty\right\}\left(\left[s_{1}\right]<\alpha,\left[s_{2}\right]<\alpha\right)$ under Condition 1.1 or Condition 1.2, where $\rho, r, \theta$ will be determined in the proof of Theorem 1.1 later.

The continuous dependence of the solution on the initial value and the decay estimate of the solution are as follows.

Theorem 1.2 Let $\varphi(x)$ and $\psi(x)$ satisfy the condition: $\|[S(t) \varphi](x)\|_{X} \leq \varepsilon$ and $\|[S(t) \psi](x)\|_{X} \leq \varepsilon, u$ and $v$ are the two solutions of problem (1.1) corresponding to initial value $\varphi(x)$ and $\psi(x)$, respectively, then

$$
\|u-v\|_{X} \leq c\|S(t)(\varphi-\psi)\|_{X}
$$

In addition, if

$$
\sup _{t>0} t^{\theta}(1+t)^{\eta}\|S(t)(\varphi-\psi)\|_{X}<+\infty, \quad \theta(\alpha+1)+\eta<1,
$$

then

$$
\|u-v\|_{X} \leq c t^{-\theta}(1+t)^{-\eta}
$$

The structure of this paper is as follows: In Sect. 2, we give an introduction to some symbols and estimates of solutions of linear equations; In Sect. 3, we give the estimates of nonlinear terms; In Sect. 4, we give the proofs of Theorem 1.1 and Theorem 1.2.

\section{Preliminary lemmas}

For the free equation

$$
\left\{\begin{array}{l}
i u_{t}+\Delta u+a \sum_{i=1}^{d} u_{x_{i} x_{i} x_{i} x_{i}}=0, \quad x \in R^{n}, t \in R, 1 \leq d<n \\
u(x, 0)=\varphi(x), \quad x \in R^{n}
\end{array}\right.
$$

by making the Fourier transformation, we obtain

$$
\left\{\begin{array}{l}
i \hat{u}_{t}-|\xi|^{2} \hat{u}+a\left(\left|\xi_{1}\right|^{4}+\left|\xi_{2}\right|^{4}+\cdots+\left|\xi_{d}\right|^{4}\right) \hat{u}=0 \\
\hat{u}(\xi, 0)=\hat{\varphi}(\xi)
\end{array}\right.
$$


Hence,

$$
\frac{d \hat{u}}{\hat{u}}=-i\left[|\xi|^{2}-a\left(\left|\xi_{1}\right|^{4}+\left|\xi_{2}\right|^{4}+\cdots+\left|\xi_{d}\right|^{4}\right)\right] d t .
$$

By substituting the initial value, we obtain

$$
\hat{u}(\xi, t)=\hat{\varphi}(\xi) e^{-i\left[|\xi|^{2}-a\left(\left|\xi_{1}\right|^{4}+\left|\xi_{2}\right|^{4}+\cdots+\left|\xi_{d}\right|^{4}\right)\right] t} .
$$

So the solution of the free equation is

$$
u(x, t)=I(x, t) * \varphi(x)=S(t) \varphi,
$$

with

$$
\begin{aligned}
I(x, t) & =\frac{1}{(2 \pi)^{n}} \int_{R^{n}} e^{i x \cdot \xi-i\left[|\xi|^{2}-a\left(\left|\xi_{1}\right|^{4}+\left|\xi_{2}\right|^{4}+\cdots+\left|\xi_{d}\right|^{4}\right)\right] t} d \xi \\
& =\frac{1}{(2 \pi)^{n}} \prod_{j=1}^{d} \int_{R} e^{i x_{j} \xi_{j}-i\left(\left|\xi_{j}\right|^{2}-a\left|\xi_{j}\right|^{4}\right) t} d \xi_{j} \prod_{j=d+1}^{n} \int_{R} e^{i x_{j} \xi_{j}-i\left|\xi_{j}\right|^{2} t} d \xi_{j} .
\end{aligned}
$$

The free equation enjoys the following time decay estimates.

Lemma 2.1 Assume that $a<0, s_{1} \geq 0, s_{2} \geq 0,2 \leq \rho<\infty, 2 \leq r<\infty$, then, for any $|t| \neq 0$, we have

$$
\|S(t) \varphi\|_{H_{\vec{y}}^{s_{1}, \rho} H_{\vec{z}}^{s_{2}, r}} \leq c|t|^{-\frac{n-d}{2}\left(1-\frac{2}{r}\right)}|t|^{-\frac{d}{4}\left(1-\frac{2}{\rho}\right)}\|\varphi\|_{H_{\vec{y}}^{s_{1}, \rho^{\prime}}} H_{\vec{z}}^{s_{2}, r^{\prime}}
$$

for $\varphi(x) \in H_{\vec{y}}^{s_{1}, \rho^{\prime}} H_{\vec{z}}^{s_{2}, r^{\prime}}$, where $r^{\prime}$ indicates the conjugate number of $r$, i.e. $\frac{1}{r}+\frac{1}{r^{\prime}}=1$.

Proof See Lemma 2.1 of [14] and Theorem 1.1 of [21], and the proof is similar to the proof of Lemma 2.2 of [19], so we omit its details.

Lemma 2.2 Assume that $f \in C^{N}(\mathcal{C})$ and $0<\mu \leq N$, where $N$ is a positive integer. Assume next that $1<p<\infty, f^{(k)}(u)=\sum_{l=0}^{k} \frac{\partial^{k} f}{\partial u^{k-l} \partial \bar{u}^{l}}(u)$. Then the following assertion hold:

(1) If $0<\mu<1$ then

$$
\left\|I^{\mu} f(u)\right\|_{p} \leq c\left\|f^{(1)}(u)\right\|_{q}\left\|I^{\mu} u\right\|_{r}
$$

where $q, r \in(1, \infty), \frac{1}{p}=\frac{1}{q}+\frac{1}{r}$.

(2) If $\mu=m$, and $m$ is a positive integer, then

$$
\left\|I^{m} f(u)\right\|_{p} \leq c \sum_{k=1}^{m}\left\|f^{(k)}(u)\right\|_{q_{k}}\left\|I^{m} u\right\|_{r_{k}}\|u\|_{s_{k}}^{k-1},
$$

where $q_{k}, r_{k} \in(1, \infty), s_{k} \in(k-1, \infty]$ and

$$
\frac{1}{p}=\frac{1}{q_{k}}+\frac{1}{r_{k}}+\frac{k-1}{s_{k}}
$$

for $k=1,2, \ldots, m$. 
(3) If $\mu=m+v$, where $m$ is a positive integer and $0<v<1$, then

$$
\left\|I^{\mu} f(u)\right\|_{p} \leq c \sum_{k=1}^{m+1}\left\|f^{(k)}(u)\right\|_{q_{k}}\left\|I^{\mu} u\right\|_{r_{k}}\|u\|_{s_{k}}^{k-1},
$$

where $q_{k}, r_{k} \in(1, \infty), s_{k} \in(k-1, \infty]$ and

$$
\frac{1}{p}=\frac{1}{q_{k}}+\frac{1}{r_{k}}+\frac{k-1}{s_{k}}
$$

for $k=1,2, \ldots, m+1$.

Proof See Lemma 3.2 of [22] and Theorem 3.1 of [23].

\section{Estimation of nonlinear terms}

According to the different values of $s_{1}, s_{2}$, the estimation of nonlinear terms can be divided into the following situations.

Lemma 3.1 Take the case $s_{1}=0$, $s_{2}=0$. Taking $2 \leq r=\alpha+2<\infty, 2 \leq \rho=\alpha+2<\infty$, we have

$$
\begin{aligned}
& \left\||u|^{\alpha} u\right\|_{L_{\vec{y}}^{\rho^{\prime}} L_{\vec{z}}^{r^{\prime}}}=\|u\|_{L_{\vec{y}}^{\rho} L_{\vec{z}}^{r}}^{\alpha+1} \\
& \left\||u|^{\alpha} u-|v|^{\alpha} v\right\|_{L_{\vec{y}}^{\rho^{\prime}}} L_{\vec{z}}^{r^{\prime}} \leq c\left(\|u\|_{L_{\vec{y}}^{\rho} L_{\vec{z}}^{r}}^{\alpha}+\|v\|_{L_{\vec{y}}^{\rho} L_{\vec{z}}^{r}}^{\alpha}\right)\|u-v\|_{L_{\vec{y}}^{\rho} L_{\vec{z}}^{r}} .
\end{aligned}
$$

Proof Firstly, since $(\alpha+1) \rho^{\prime}=\rho,(\alpha+1) r^{\prime}=r$ we have

$$
\left\||u|^{\alpha} u\right\|_{L_{\vec{y}}^{\rho^{\prime}} L_{\vec{z}}^{r^{\prime}}}=\|u\|_{L_{\vec{y}}^{(\alpha+1) \rho^{\prime}}}^{\alpha+1} L_{\bar{z}}^{(\alpha+1) r^{\prime}}=\|u\|_{L_{\vec{y}}^{\rho} L_{\vec{z}}^{r}}^{\alpha+1}
$$

which completes the proof of the first inequality.

Secondly, since $\left.|| u\right|^{\alpha} u-|v|^{\alpha} v\left|\leq c\left(|u|^{\alpha}+|v|^{\alpha}\right)\right| u-v \mid$, by using the Hölder inequality we have

$$
\begin{aligned}
& \left\||u|^{\alpha} u-|v|^{\alpha} v\right\|_{L_{\vec{y}}^{\rho^{\prime}}} L_{\vec{z}}^{r^{\prime}} \\
& \leq c\left\||u|^{\alpha}+|v|^{\alpha}\right\|_{L_{\vec{y}}^{\rho_{1}} L_{\vec{z}}^{r_{1}}}\|u-v\|_{L_{\vec{y}}^{\rho} L_{\vec{z}}^{r}} \\
& \leq c\left(\|u\|_{L_{\vec{y}}^{\rho_{1} \alpha} L_{\vec{z}}^{r_{1} \alpha}}^{\alpha}+\|v\|_{L_{\vec{y}}^{\rho_{1} \alpha} L_{\vec{z}}^{r_{1} \alpha}}^{\alpha}\right)\|u-v\|_{L_{\vec{y}}^{\rho} L_{\vec{z}}^{r}} \\
& \quad \leq c\left(\|u\|_{L_{\vec{y}}^{\rho} L_{\vec{z}}^{r}}^{\alpha}+\|v\|_{L_{\vec{y}}^{\rho} L_{\vec{z}}^{r}}^{\alpha}\right)\|u-v\|_{L_{\vec{y}}^{\rho} L_{\vec{z}}^{r},},
\end{aligned}
$$

where $1 / \rho^{\prime}=1 / \rho_{1}+1 / \rho, 1 / r^{\prime}=1 / r_{1}+1 / r, \rho_{1} \alpha=\rho, r_{1} \alpha=r$.

For the cases $s_{1} \neq 0, s_{2} \neq 0\left(\left[s_{1}\right]<\alpha,\left[s_{2}\right]<\alpha\right)$, using the fractional Sobolev embedding ([24]), we can obtain the following lemmas. 
Lemma 3.2 Take the case $0<s_{1}<\frac{d}{2}, s_{2}>\frac{n-d}{2}$. Taking $\rho=\frac{d(\alpha+2)}{d+s_{1} \alpha}, r=2$, we have

$$
\begin{aligned}
& \left\||u|^{\alpha} u\right\|_{H_{\vec{y}}^{s_{1}, \rho^{\prime}}} H_{\vec{z}}^{s_{2}, r^{\prime}} \leq c\|u\|_{H_{\vec{y}}^{s_{1}, \rho}}^{\alpha+1} H_{\vec{z}}^{s_{2}, r}, \\
& \left\||u|^{\alpha} u-|v|^{\alpha} v\right\|_{L_{\vec{y}}^{\rho^{\prime}}} L_{\vec{z}}^{r^{\prime}} \leq c\left(\|u\|_{H_{\vec{y}}^{s_{1}, \rho}}^{\alpha} H_{\vec{z}}^{s_{2}, r}+\|v\|_{H_{\vec{y}}^{s_{1}, \rho}}^{\alpha} H_{\vec{z}}^{s_{2}, r},\|u-v\|_{L_{\vec{y}}^{\rho} L_{\vec{z}}^{r}} .\right.
\end{aligned}
$$

Proof Using the Sobolev embedding $H_{\vec{z}}^{s_{2}, r}\left(R^{n-d}\right) \hookrightarrow L_{\vec{z}}^{\infty}\left(R^{n-d}\right)$ we have

$$
\|u\|_{L_{\vec{z}}^{\infty}\left(R^{n-d}\right)} \leq c\|u\|_{H_{\vec{z}}^{s, r}\left(R^{n-d}\right)}
$$

By the Hölder inequality we obtain

$$
\begin{aligned}
\left\||u|^{\alpha+1}\right\|_{H_{\vec{z}}^{s_{2}, r^{\prime}}} & \leq\left\||u|^{\alpha+1}\right\|_{L_{\vec{z}}^{r^{\prime}}}+\left\|\nabla_{\vec{z}}^{s_{2}}\left(|u|^{\alpha+1}\right)\right\|_{L_{\vec{z}}^{r^{\prime}}} \\
& \leq c\|u\|_{L_{\vec{z}}^{\infty}}^{\alpha}\|u\|_{L_{\vec{z}}^{r}}+c\|u\|_{L_{\vec{z}}^{\infty}}^{\alpha}\left\|\nabla_{\vec{z}}^{s_{2}} u\right\|_{L_{\vec{z}}^{r}} \\
& \leq c\|u\|_{L_{\vec{z}}^{\infty}}^{\alpha}\|u\|_{H_{\vec{z}}^{s_{2}, r}} \\
& \leq c\|u\|_{H_{\vec{z}}^{s_{2}, r}}^{\alpha+1}
\end{aligned}
$$

where $\frac{1}{r^{\prime}}=\frac{1}{\infty}+\frac{1}{r}$ since $r=2$.

Therefore we obtain

$$
\begin{aligned}
& \left\||u|^{\alpha+1}\right\|_{H_{\vec{y}}^{s_{1}, \rho^{\prime}}} H_{\vec{z}}^{s_{2}, r^{\prime}}=\|\||u|^{\alpha+1}\left\|_{H_{\vec{z}}^{s_{2}, r^{\prime}}}\right\|_{H_{\vec{y}}^{s_{1}, \rho^{\prime}}} \\
& \leq c\|\| u\left\|_{H_{\vec{z}}^{s 2, r}}^{\alpha+1}\right\|_{H_{\vec{y}}^{s_{1}, \rho^{\prime}}} \\
& =c\left\|f\left(\|u\|_{H_{\vec{z}}^{s_{2}}, r}\right)\right\|_{H_{\vec{y}}^{s_{1}, \rho^{\prime}}} \\
& \leq c\left\|f\left(\|u\|_{H_{\vec{z}}^{s_{2}, r}}\right)\right\|_{L_{\vec{y}}^{\rho^{\prime}}}+c\left\|\nabla_{\vec{y}}^{s_{1}} f\left(\|u\|_{H_{\vec{z}}^{s_{2}, r}}\right)\right\|_{L_{\vec{y}}^{\rho^{\prime}}} \\
& \leq c\|\| u\left\|_{H_{\vec{z}}^{s_{2}}, r}^{\alpha}\right\|_{L_{\vec{y}}^{\frac{q}{\alpha}}}\|\| u\left\|_{H_{\vec{z}}^{s_{2}, r}}\right\|_{L_{\vec{y}}^{\rho}}+c\|\| u\left\|_{H_{\vec{z}}^{s_{2}}, r}^{\alpha}\right\|_{L_{\vec{y}}^{\frac{q}{\alpha}}}\left\|\nabla_{\vec{y}}^{s_{1}}\left(\|u\|_{H_{\vec{z}}^{s_{2}, r}}\right)\right\|_{L_{\vec{y}}^{\rho}} \\
& \leq c\|\| u\left\|_{H_{\vec{z}}^{s_{2}, r}}\right\|_{L_{\vec{y}}^{q}}^{\alpha}\|\| u\left\|_{H_{\vec{z}}^{s_{2}}, r}\right\|_{L_{\vec{y}}^{\rho}}+c\|\| u\left\|_{H_{\vec{z}}^{s_{2}}, r}\right\|_{L_{\vec{y}}^{q}}^{\alpha}\left\|\nabla_{\vec{y}}^{s_{1}}\left(\|u\|_{H_{\vec{z}}^{s_{2}, r}}\right)\right\|_{L_{\vec{y}}^{\rho}} \\
& \leq c\|\| u\left\|_{H_{\vec{z}}^{s_{2}, r}}\right\|_{L_{\vec{y}}^{q}}^{\alpha}\|\| u\left\|_{H_{\vec{z}}^{s_{2}, r}}\right\|_{H_{\vec{y}}^{s_{1}, \rho}} \\
& \leq c\|u\|_{H_{\vec{y}}^{s_{1}, \rho} H_{\vec{z}}^{s_{2}, r}}^{\alpha+1}
\end{aligned}
$$

where $f(z)=z^{\alpha+1}, \frac{1}{\rho^{\prime}}=\frac{1}{\frac{q}{\alpha}}+\frac{1}{\rho}$. Meanwhile, we use the Sobolev embedding $H_{\vec{y}}^{s_{1}, \rho}\left(R^{d}\right) \hookrightarrow$ $L_{\vec{y}}^{q}\left(R^{d}\right), \frac{1}{q}=\frac{1}{\rho}-\frac{s_{1}}{d}$ and $s_{1}<\frac{d}{\rho}$.

Using the Hölder inequality and the Sobolev embedding $H_{\vec{y}}^{s_{1}, \rho}\left(R^{d}\right) \hookrightarrow L_{\vec{y}}^{\rho_{1}}\left(R^{d}\right), \frac{1}{\rho_{1}}=\frac{1}{\rho}-$ $\frac{s_{1}}{d}$, we have

$$
\begin{aligned}
& \left\||u|^{\alpha} u-|v|^{\alpha} v\right\|_{L_{\vec{y}}^{\rho^{\prime}}} L_{\vec{z}}^{r^{\prime}} \\
& \quad \leq c\left(\|u\|_{L_{\vec{y}}^{\rho_{1}} L_{\vec{z}}^{r_{1}}}^{\alpha}+\|v\|_{L_{\vec{y}}^{\rho_{1}} L_{\vec{z}}^{r_{1}}}^{\alpha}\right)\|u-v\|_{L_{\vec{y}}^{\rho}} L_{\vec{z}}^{r}
\end{aligned}
$$




$$
\begin{aligned}
& \leq c\left(\|u\|_{L_{\bar{y}}^{\rho 1} L_{\bar{z}}^{\infty}}^{\alpha}+\|v\|_{L_{\bar{y}}^{\rho_{1}} L_{\bar{z}}^{\infty}}^{\alpha}\right)\|u-v\|_{L_{\tilde{y}}^{\rho} L_{\bar{z}}^{r}} \\
& \leq c\left(\|u\|_{H_{\vec{y}}^{s_{1}, \rho} H_{\bar{z}}^{s_{2}, r}}^{\alpha}+\|v\|_{H_{\bar{y}}^{s_{1}, \rho} H_{\bar{z}}^{s_{2}}, r}^{\alpha}\right)\|u-v\|_{L_{\tilde{y}}^{\rho} L_{\vec{z}}^{r}},
\end{aligned}
$$

where $1 / \rho^{\prime}=\alpha / \rho_{1}+1 / \rho, 1 / r^{\prime}=\alpha / r_{1}+1 / r$.

Lemma 3.3 Take the case $s_{1}>\frac{d}{2}, 0<s_{2}<\frac{n-d}{2}$. Taking $\rho=2, r=\frac{(\alpha+2)(n-d)}{n-d+\alpha s_{2}}$, we have

$$
\begin{aligned}
& \left\||u|^{\alpha} u\right\|_{H_{\vec{y}}^{s_{1}}, \rho^{\prime}} H_{\bar{z}}^{s_{2}, r^{\prime}} \leq c\|u\|_{H_{\bar{y}}^{s_{1}, \rho}}^{\alpha+1} H_{\bar{z}}^{s_{2}, r}, \\
& \left\||u|^{\alpha} u-|v|^{\alpha} v\right\|_{L_{\bar{y}}^{\rho^{\prime}} L_{\bar{z}}^{r^{\prime}}} \leq c\left(\|u\|_{H_{\bar{y}}^{s_{1}, \rho} H_{\bar{z}}^{s_{2}, r}+\|v\|_{H_{\bar{y}}^{s_{1}, \rho}}^{\alpha} H_{\bar{z}}^{s_{2}, r}}^{\alpha}\right)\|u-v\|_{L_{\vec{y}}^{\rho} L_{\vec{z}}^{r} .}
\end{aligned}
$$

Proof In a similar way as in Lemma 3.2: using the Sobolev embedding $H_{\vec{z}}^{s_{2}, r}\left(R^{n-d}\right) \hookrightarrow$ $L_{\vec{z}}^{q}\left(R^{n-d}\right), \frac{1}{q}=\frac{1}{r}-\frac{s_{2}}{n-d}$ and $H_{\vec{y}}^{s_{1}, \rho}\left(R^{d}\right) \hookrightarrow L_{\vec{y}}^{\infty}\left(R^{d}\right)$, one obtains the proof of the first inequality. Using the Hölder inequality and the Sobolev embedding $H_{\vec{z}}^{s_{2}, r}\left(R^{n-d}\right) \hookrightarrow L_{\vec{z}}^{r_{1}}\left(R^{n-d}\right)$, $\frac{1}{r_{1}}=\frac{1}{r}-\frac{s_{2}}{n-d}$, one obtains the proof of the second inequality.

Lemma 3.4 Take the case $s_{1}=\frac{d}{2}$.

(1) When $0<s_{2}<\frac{n-d}{2}$, taking $\rho=\alpha+2, r=\frac{(\alpha+2)(n-d)}{n-d+\alpha s_{2}}$, we have

$$
\begin{aligned}
& \left\||u|^{\alpha} u\right\|_{H_{\bar{y}}^{s_{1}}, \rho^{\prime}} H_{\bar{z}}^{s_{2}, r^{\prime}} \leq c\|u\|_{H_{\bar{y}}^{s_{1}, \rho}}^{\alpha+1} H_{\bar{z}}^{s_{2}, r}, \\
& \left\||u|^{\alpha} u-|v|^{\alpha} v\right\|_{L_{\vec{y}}^{\rho^{\prime}} H_{\bar{z}}^{s_{2}, r^{\prime}}} \leq c\left(\|u\|_{L_{\vec{y}}^{\rho} H_{\vec{z}}^{\delta_{2}, r}}^{\alpha}+\|v\|_{L_{\vec{y}}^{\rho} H_{\vec{z}}^{s_{2}, r}}^{\alpha}\|u-v\|_{L_{\vec{y}}^{\rho} H_{\bar{z}}^{s_{2}, r}} .\right.
\end{aligned}
$$

(2) When $s_{2}>\frac{n-d}{2}$, taking $\rho=\alpha+2, r=2$, we have

$$
\begin{aligned}
& \left\||u|^{\alpha} u\right\|_{H_{\bar{y}}^{s_{1}, \rho^{\prime}}} H_{\vec{z}}^{s_{2}, r^{\prime}} \leq c\|u\|_{H_{\bar{y}}^{s_{1}, \rho}}^{\alpha+1} H_{\vec{z}}^{s_{2}, r}, \\
& \left\||u|^{\alpha} u-|v|^{\alpha} v\right\|_{L_{\vec{y}}^{\rho^{\prime}} H_{\vec{z}}^{s_{2}, r^{\prime}}} \leq c\left(\|u\|_{L_{\vec{y}}^{\rho} H_{\vec{z}}^{s_{2}, r}}^{\alpha}+\|v\|_{L_{\vec{y}}^{\rho} H_{\vec{z}}^{s_{2}, r}}^{\alpha}\right)\|u-v\|_{L_{\vec{y}}^{\rho} H_{\vec{z}}^{s_{2}, r}}
\end{aligned}
$$

Proof (1) Using the Sobolev embedding $H_{\bar{z}}^{s_{2}, r}\left(R^{n-d}\right) \hookrightarrow L_{\bar{z}}^{q}\left(R^{n-d}\right), \frac{1}{q}=\frac{1}{r}-\frac{s_{2}}{n-d}$, we have

$$
\|u\|_{L_{\bar{z}}^{q}\left(R^{n-d}\right)} \leq c\|u\|_{H_{\bar{z}}^{s_{2}, r_{2}}\left(R^{n-d}\right)}
$$

Using the Hölder inequality we have

$$
\begin{aligned}
\left\||u|^{\alpha} u\right\|_{H_{\bar{z}}^{s_{2}, r^{\prime}}} & =\left\||u|^{\alpha} u\right\|_{L_{\bar{z}}^{r^{\prime}}}+\left\|\nabla_{\vec{z}}^{s_{2}}\left(|u|^{\alpha} u\right)\right\|_{L_{\frac{z}{z}}^{r^{\prime}}} \\
& \leq c\left\||u|^{\alpha}\right\|_{L_{\bar{z}}^{\alpha}}\|u\|_{L_{\bar{z}}^{r}}+c\left\||u|^{\alpha}\right\|_{L_{\bar{z}}^{\frac{q}{\alpha}}}\left\|\nabla_{\bar{z}}^{s_{2}} u\right\|_{L_{\bar{z}}^{r}} \\
& \leq c\|u\|_{L_{\bar{z}}^{q}}^{\alpha}\|u\|_{H_{\bar{z}}^{s_{2}, r}} \\
& \leq c\|u\|_{H_{\bar{z}}^{\alpha, r}, r}^{\alpha+1},
\end{aligned}
$$

where $\frac{1}{r^{\prime}}=\frac{1}{\frac{q}{\alpha}}+\frac{1}{r}$, which means $r=\frac{(\alpha+2)(n-d)}{n-d+\alpha s_{2}}$. 
We have

$$
\begin{aligned}
& \left\||u|^{\alpha} u\right\|_{H_{y}^{s_{1}, \rho^{\prime}} H_{\bar{z}}^{s_{2}, r^{\prime}}}=\|\||u|^{\alpha} u\left\|_{H_{\bar{z}}^{s_{2}, r^{\prime}}}\right\|_{H_{y}^{s_{1}}, \rho^{\prime}} \\
& \leq c\|\| u\left\|_{H_{\bar{z}}^{s_{2}, r}}^{\alpha+1}\right\|_{H_{\vec{y}}^{s_{1}, \rho^{\prime}}} \\
& =c\left\|f\left(\|u\|_{H_{\bar{z}}^{s_{2}, r}}\right)\right\|_{H_{\bar{y}}^{s_{1}, \rho^{\prime}}} \\
& \leq c \sum_{k=1}^{m}\left\|f^{(k)}\left(\|u\|_{H_{\bar{z}}^{s_{2}, r}}\right)\right\|_{L_{\bar{y}}^{q_{k}}}\left\|I^{s_{1}}\right\| u\left\|_{H_{\bar{z}}^{s_{2}, r}}\right\|_{L_{\tilde{y}}^{l_{k}}}\|\| u\left\|_{H_{\bar{z}}^{s_{2}, r}}\right\|_{L_{\tilde{y}}^{m_{k}}}^{k-1} \\
& \leq c \sum_{k=1}^{m}\|\| u\left\|_{H_{\bar{z}}^{2}, r, r}^{\alpha+1-k}\right\|_{L_{\tilde{y}}^{q_{k}}}\left\|I^{s_{1}}\right\| u\left\|_{H_{\bar{z}}^{s_{2}, r}}\right\|_{L_{\tilde{y}}^{l_{k}}}\|\| u\left\|_{H_{\bar{z}}^{s_{2}, r}}\right\|_{L_{\vec{y}}^{m}}^{k-1} \\
& \leq c \sum_{k=1}^{m}\|\| u\left\|_{H_{\tilde{z}}^{s_{2}, r}}\right\|_{L_{\vec{y}}^{\alpha+1-k) q_{k}}}^{\alpha+1-k}\left\|I^{s_{1}}\right\| u\left\|_{H_{\tilde{z}}^{s_{2}, r}}\right\|_{L_{\vec{y}}^{l_{k}}}\|\| u\left\|_{H_{\tilde{z}}^{s_{2}, r}}\right\|_{L_{\vec{y}}^{m_{k}}}^{k-1} \\
& \leq c \sum_{k=1}^{m}\|\| u\left\|_{H_{\bar{z}}^{s_{2}, r}}\right\|_{L_{\vec{y}}^{m_{k}}}^{\alpha+1-k}\|u\|_{H_{\vec{y}}^{s_{1}, l_{k}} H_{\bar{z}}^{s_{2}, r}}\|\| u\left\|_{H_{\vec{z}}^{s_{2}, r}}\right\|_{L_{\vec{y}}^{m_{k}}}^{k-1} \\
& \leq c \sum_{k=1}^{m}\|\| u\left\|_{H_{\bar{z}}^{s_{2}, r}}\right\|_{L_{\vec{y}}^{\rho}}^{\alpha}\|u\|_{H_{\tilde{y}}^{s_{1}, \rho} H_{\bar{z}}^{s_{2}, r}} \\
& \leq c\|\| u\left\|_{H_{\bar{z}}^{s_{2}, r}}\right\|_{L_{\bar{y}}^{\rho}}^{\alpha}\|u\|_{H_{\bar{y}}^{s_{1}, \rho}} H_{\bar{z}}^{s_{2}, r} \\
& \leq c\|u\|_{H_{\bar{y}}^{s} 1, \rho}^{\alpha+1} H_{z}^{s_{2}, r},
\end{aligned}
$$

where $f$ is the same as before. When $0<d / 2<1$, let $m=1$; when $d / 2=[d / 2]$, let $m=$ $[d / 2]$; when $d / 2=[d / 2]+\sigma(0<\sigma<1)$, let $m=[d / 2]+1$. For the index $\frac{1}{\rho^{\prime}}=\frac{1}{q_{k}}+\frac{1}{l_{k}}+\frac{k-1}{m_{k}}$, $(\alpha+1-k) q_{k}=m_{k}=\rho, l_{k}=\rho$, which means $\rho=\alpha+2$.

And

$$
\begin{aligned}
\left\||u|^{\alpha} u-|v|^{\alpha} v\right\|_{H_{\bar{z}}^{s_{2}, r^{\prime}}} & \\
\leq & c\left\|\left(|u|^{\alpha}+|v|^{\alpha}\right)|u-v|\right\|_{H_{\bar{z}}^{s_{2}, r^{\prime}}} \\
\leq & c\left(\left\||u|^{\alpha}+|v|^{\alpha}\right\|_{H_{\bar{z}}^{s_{2}, p_{1}}}\|u-v\|_{L_{\bar{z}}^{p_{2}}}+\left\||u|^{\alpha}+|v|^{\alpha}\right\|_{L \bar{z}}^{p_{3}}\|u-v\|_{H_{\bar{z}}^{s_{2}, p_{4}}}\right) \\
\leq & c\left[\left(\|u\|_{L_{\bar{z}}^{a_{1}(\alpha-1)}}^{\alpha-1}\|u\|_{H_{\bar{z}}^{s_{2}, b_{1}}}+\|v\|_{L_{\bar{z}}^{a_{1}(\alpha-1)}}^{\alpha-1}\|v\|_{H_{\bar{z}}^{s_{2}, b_{1}}}\right)\|u-v\|_{H_{\bar{z}}^{s_{2}, r}}\right. \\
& \left.+\left(\|u\|_{L_{\bar{z}}^{\alpha p_{3}}}^{\alpha}+\|v\|_{L_{\bar{z}}^{\alpha p_{3}}}^{\alpha}\right)\|u-v\|_{H_{\bar{z}}^{s_{2}, p p_{4}}}\right] \\
\leq & c\left[\left(\|u\|_{H_{\bar{z}}^{s_{2}, r}}^{\alpha}+\|v\|_{H_{\bar{z}}^{s_{2}, r}}^{\alpha}\right)\|u-v\|_{H_{\bar{z}}^{s_{2}, r}}+\left(\|u\|_{H_{\bar{z}}^{s_{2}, r}}^{\alpha}+\|v\|_{H_{\bar{z}}^{s_{2}, r}}^{\alpha}\right)\|u-v\|_{H_{\bar{z}}^{s_{2}, p_{4}}}\right] \\
\leq & c\left(\|u\|_{H_{\bar{z}}^{s_{2}, r}}^{\alpha}+\|v\|_{H_{\bar{z}}^{s_{2}, r}}^{\alpha}\right)\|u-v\|_{H_{\bar{z}}^{s_{2}, r},}
\end{aligned}
$$

where $\frac{1}{r^{\prime}}=\frac{1}{p_{1}}+\frac{1}{p_{2}}=\frac{1}{p_{3}}+\frac{1}{p_{4}}, 1 / p_{1}=1 / a_{1}+1 / b_{1}, 1 / a_{1}(\alpha-1)=1 / r-s_{2} /(n-d), b_{1}=r, 1 / p_{2}=$ $1 / r-s_{2} /(n-d), 1 /\left(\alpha p_{3}\right)=1 / r-s_{2} /(n-d), p_{4}=r$. Therefore,

$$
\begin{aligned}
& \left\||u|^{\alpha} u-|v|^{\alpha} v\right\|_{L_{\vec{y}}^{\rho^{\prime}} H_{\vec{z}}^{s_{2}, r^{\prime}}} \\
& \quad \leq c\|\||u|^{\alpha} u-|v|^{\alpha} v\left\|_{H_{\vec{z}}^{s_{2}, r^{\prime}}}\right\|_{L_{\vec{y}}^{\rho^{\prime}}}
\end{aligned}
$$




$$
\begin{aligned}
& \leq c\left\|\left(\|u\|_{H_{\bar{z}}^{s_{2}, r}}^{\alpha}+\|v\|_{H_{\bar{z}}^{s_{2}, r}}^{\alpha}\right)\right\| u-v\left\|_{H_{\bar{z}}^{s_{2}, r}}\right\|_{L_{\vec{y}}^{\rho^{\prime}}} \\
& \leq c\|\| u\left\|_{H_{\bar{z}}^{s_{2}, r}}^{\alpha}+\right\| v\left\|_{H_{\bar{z}}^{s_{2}}, r}^{\alpha}\right\|_{L_{\vec{y}}^{\frac{p_{1}}{\alpha}}}\|\| u-v\left\|_{H_{\bar{z}}^{s_{2}}, r}\right\|_{L_{\bar{y}}^{\rho}} \\
& \leq c\left(\|u\|_{L_{\vec{y}}^{p_{1}} H_{\bar{z}}^{s_{2}, r}}^{\alpha}+\|v\|_{L_{\bar{y}}^{p_{1}} H_{\bar{z}}^{s_{2}, r}}^{\alpha}\right)\|u-v\|_{L_{\vec{y}}^{\rho} H_{\bar{z}}^{s_{2}, r}} \\
& \leq c\left(\|u\|_{L_{\vec{y}}^{\rho} H_{\bar{z}}^{s_{2}, r}}^{\alpha}+\|v\|_{L_{\vec{y}}^{\rho} H_{\tilde{z}}^{\delta_{2}, r}}^{\alpha}\right)\|u-v\|_{L_{\vec{y}}^{\rho} H_{\bar{z}}^{\delta_{2}}, r,},
\end{aligned}
$$

where $\frac{1}{\rho^{\prime}}=\frac{\alpha}{p_{1}}+\frac{1}{\rho}, p_{1}=\rho$.

(2) Similarly, using the Sobolev embedding $H_{\vec{z}}^{s_{2}, r_{2}}\left(R^{n-d}\right) \hookrightarrow L_{\vec{z}}^{\infty}\left(R^{n-d}\right)$, we get the conclusion.

Lemma 3.5 Take the case $s_{2}=\frac{n-d}{2}$.

(1) When $0<s_{1}<\frac{d}{2}$, taking $\rho=\frac{d(\alpha+2)}{d+s_{1} \alpha}, r=\alpha+2$, we have

$$
\begin{aligned}
& \left\||u|^{\alpha} u\right\|_{H_{\bar{y}}^{s_{1}}, \rho^{\prime}} H_{\bar{z}}^{s_{2}, r^{\prime}} \leq c\|u\|_{H_{\bar{y}}^{s_{1}, \rho}}^{\alpha+1} H_{\bar{z}}^{s_{2}, r},
\end{aligned}
$$

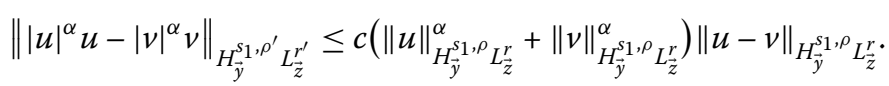

(2) When $s_{1}>\frac{d}{2}$, taking $\rho=2, r=\alpha+2$, we have

$$
\begin{aligned}
& \left\||u|^{\alpha} u\right\|_{H_{\bar{y}}^{s_{1}, \rho^{\prime}} H_{\bar{z}}^{s_{2}, r^{\prime}}} \leq c\|u\|_{H_{\bar{y}}^{s_{1}}, \rho}^{\alpha+\rho} H_{\bar{z}}^{s_{2}, r}, \\
& \left\||u|^{\alpha} u-|v|^{\alpha} v\right\|_{H_{\bar{y}}^{s_{1}, \rho^{\prime}} L_{\bar{z}}^{r^{\prime}}} \leq c\left(\|u\|_{H_{\bar{y}}^{s_{1}, \rho} L_{\bar{z}}^{r}}^{\alpha}+\|v\|_{H_{\bar{y}}^{s_{1}, \rho} L_{\bar{z}}^{r}}^{\alpha}\|u-v\|_{H_{\bar{y}}^{s_{1}, \rho} L_{\bar{z}}^{r} .}\right.
\end{aligned}
$$

Proof In a similar way to Lemma 3.4, using the Sobolev embedding $H_{\vec{y}}^{s_{1}, \rho}\left(R^{d}\right) \hookrightarrow L_{\vec{y}}^{q}\left(R^{d}\right)$, $\frac{1}{q}=\frac{1}{\rho}-\frac{s_{1}}{d}$ and $H_{\vec{y}}^{s_{1}, \rho}\left(R^{d}\right) \hookrightarrow L_{\vec{y}}^{\infty}\left(R^{d}\right)$, we have the above lemma.

Lemma 3.6 Take the case $s_{1}=\frac{d}{2}, s_{2}=\frac{n-d}{2}$. Taking $\rho=\alpha+2, r=\alpha+2$, we have

$$
\begin{aligned}
& \left\||u|^{\alpha} u\right\|_{H_{\vec{y}}^{s_{1}, \rho^{\prime}}} H_{\bar{z}}^{s_{2}, r^{\prime}} \leq c\|u\|_{H_{\bar{y}}^{s_{1}, \rho} H_{\vec{z}}^{s_{2}, r}}^{\alpha,},
\end{aligned}
$$

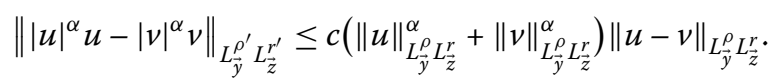

Proof By Lemma 2.2 we have

$$
\begin{aligned}
\left\||u|^{\alpha} u\right\|_{H_{\bar{z}}^{s_{2}, r^{\prime}}} & \leq c \sum_{k=1}^{m}\left\|g^{(k)}(u)\right\|_{L_{\bar{z}}^{q_{k}}}\left\|I^{s_{2}} u\right\|_{L_{\bar{z}}^{l_{k}}}\|u\|_{L_{\bar{z}}^{m_{k}}}^{k-1} \\
& \leq c \sum_{k=1}^{m}\left\|u^{\alpha+1-k}\right\|_{L_{\bar{z}}^{q_{k}}}\left\|I^{s_{2}} u\right\|_{L_{\bar{z}}^{l_{k}}}\|u\|_{L_{\bar{z}}^{m}}^{k-1} \\
& \leq c \sum_{k=1}^{m}\|u\|_{L_{\bar{z}}^{(\alpha+1-k) q_{k}}}^{\alpha+1-k}\|u\|_{H_{\bar{z}}^{s_{2}, l_{k}}}\|u\|_{L_{\bar{z}}^{m_{k}}}^{k-1} \\
& \leq c\|u\|_{L_{\bar{z}}^{m_{k}}}^{\alpha}\|u\|_{H_{\bar{z}}^{s_{2}, l_{k}}} \\
& \leq c\|u\|_{L_{\bar{z}}^{r}}^{\alpha}\|u\|_{H_{\bar{z}}^{s_{2}, l_{k}}}
\end{aligned}
$$




$$
\begin{aligned}
& \leq c\|u\|_{H_{\bar{z}}^{s_{2}, r}}^{\alpha}\|u\|_{H_{\bar{z}}^{s_{2}, r}} \\
& \leq c\|u\|_{H_{\bar{z}}^{s_{2}, r}}^{\alpha+1},
\end{aligned}
$$

where $g(u)=|u|^{\alpha} u$. When $0<(n-d) / 2<1$, let $m=1$; when $(n-d) / 2=[(n-d) / 2]$, let $m=[(n-d) / 2]$; when $(n-d) / 2=[(n-d) / 2]+\sigma(0<\sigma<1)$, let $m=[(n-d) / 2]+1$. For the index $\frac{1}{r^{\prime}}=\frac{1}{q_{k}}+\frac{1}{l_{k}}+\frac{k-1}{m_{k}},(\alpha+1-k) q_{k}=m_{k}=r, l_{k}=r$, then $r=\alpha+2$ and

$$
\begin{aligned}
& \left\||u|^{\alpha} u\right\|_{H_{\bar{y}}^{s_{1}, \rho^{\prime}}} H_{\bar{z}}^{s_{2}, r^{\prime}}=\|\||u|^{\alpha} u\left\|_{H_{\bar{z}}^{s_{2}, r^{\prime}}}\right\|_{H_{\bar{y}}^{s_{1}, \rho^{\prime}}} \\
& \leq c\|\| u\left\|_{H_{\bar{z}}^{s_{2}, r}}^{\alpha+1}\right\|_{H_{\vec{y}}^{s_{1}}, \rho^{\prime}} \\
& \leq c \sum_{k=1}^{m}\left\|f^{(k)}\left(\|u\|_{H_{\bar{z}}^{s_{2}, r}}\right)\right\|_{L_{\tilde{y}}^{q_{k}}}\left\|I^{s_{1}}\right\| u\left\|_{H_{\bar{z}}^{s_{2}, r}}\right\|_{L_{\bar{y}}^{l_{k}}}\|\| u\left\|_{H_{\bar{z}}^{s_{2}, r}}\right\|_{L_{\tilde{y}}^{m_{k}}}^{k-1} \\
& \leq c \sum_{k=1}^{m}\|\| u\left\|_{H_{\tilde{z}}^{2}, r}^{\alpha+1-k}\right\|_{L_{\vec{y}}^{q_{k}}}\left\|I^{s_{1}}\right\| u\left\|_{H_{\bar{z}}^{s_{2}, r}}\right\|_{L_{\tilde{y}}^{l_{k}}}\|\| u\left\|_{H_{\tilde{z}}^{s_{2}, r}}\right\|_{L_{\vec{y}}^{m_{k}}}^{k-1} \\
& \leq c \sum_{k=1}^{m}\|\| u\left\|_{H_{\vec{z}}^{s_{2}, r}}\right\|_{L_{\vec{y}}^{(\alpha+1-k) q_{k}}}^{\alpha+1-k}\left\|I^{s_{1}}\right\| u\left\|_{H_{\bar{z}}^{s_{2}, r}}\right\|_{L_{\vec{y}}^{l_{k}}}\|\| u\left\|_{H_{\vec{z}}^{s_{2}, r}}\right\|_{L_{\vec{y}}^{m_{k}}}^{k-1}
\end{aligned}
$$

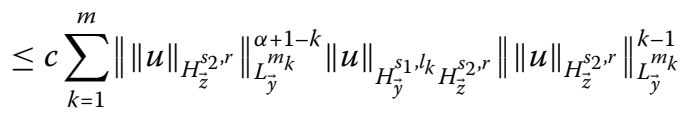

$$
\begin{aligned}
& \leq c \sum_{k=1}^{m}\|\| u\left\|_{H_{\vec{z}}^{s_{2}, r}}\right\|_{L_{\vec{y}}^{m} k}^{\alpha}\|u\|_{H_{\vec{y}}^{s_{1}, l_{k}} H_{\vec{z}}^{s_{2}, r}} \\
& \leq c\|\| u\left\|_{H_{\bar{z}}^{s_{2}}, r}\right\|_{L_{\bar{y}}^{\rho}}^{\alpha}\|u\|_{H_{\bar{y}}^{s_{1}, \rho} H_{\bar{z}}^{s_{2}, r}} \\
& \leq c\|u\|_{H_{\bar{y}}^{s_{1}, \rho}}^{\alpha+s_{z}, r, r},
\end{aligned}
$$

where $f$ is the same as before. When $0<d / 2<1$, let $m=1$; when $d / 2=[d / 2]$, let $m=$ $[d / 2]$; when $d / 2=[d / 2]+\sigma(0<\sigma<1)$ let $m=[d / 2]+1$. For the index $\frac{1}{\rho^{\prime}}=\frac{1}{q_{k}}+\frac{1}{l_{k}}+\frac{k-1}{m_{k}}$, $(\alpha+1-k) q_{k}=m_{k}=\rho, l_{k}=\rho$, then $\rho=\alpha+2$ and we have $\frac{1}{\rho^{\prime}}=\frac{\alpha}{\rho}+\frac{1}{\rho}, \frac{1}{r^{\prime}}=\frac{\alpha}{r}+\frac{1}{r}$.

In the same way, using the Hölder inequality we have

$$
\left\||u|^{\alpha} u-|v|^{\alpha} v\right\|_{L_{\bar{y}}^{\rho^{\prime}} L_{\bar{z}}^{r^{\prime}}} \leq c\left(\|u\|_{L_{\tilde{y}}^{\rho} L_{\tilde{z}}^{r}}^{\alpha}+\|v\|_{L_{\tilde{y}}^{\rho} L_{\tilde{z}}^{r}}^{\alpha}\right)\|u-v\|_{L_{\tilde{y}}^{\rho} L_{\tilde{z}}^{r}}
$$

Lemma 3.7 For the case $0 \leq s_{1}<\frac{d}{2}, 0 \leq s_{2}<\frac{n-d}{2}$, taking $\rho=\frac{d(\alpha+2)}{d+s_{1} \alpha}, r=\frac{(\alpha+2)(n-d)}{n-d+\alpha s_{2}}$, we have

$$
\begin{aligned}
& \left\||u|^{\alpha} u\right\|_{H_{\bar{y}}^{s_{1}, \rho^{\prime}} H_{\vec{z}}^{s_{2}, r^{\prime}}} \leq c\|u\|_{H_{\bar{y}}^{s_{1}, \rho} H_{\vec{z}}^{s_{2}, r}}^{\alpha+1}, \\
& \left\||u|^{\alpha} u-|v|^{\alpha} v\right\|_{L_{\vec{y}}^{\rho^{\prime}} L_{\bar{z}}^{r^{\prime}}} \leq c\left(\|u\|_{H_{\vec{y}}^{s_{1}}, \rho}^{\alpha} H_{\bar{z}}^{s_{2}, r}+\|v\|_{H_{\bar{y}}^{s_{1}, \rho} H_{\bar{z}}^{s_{2}, r}}^{\alpha}\right)\|u-v\|_{L_{\tilde{y}}^{\rho} L_{\tilde{z}}^{r}} .
\end{aligned}
$$

Proof Using the Sobolev embedding $H_{\vec{z}}^{s_{2}, r}\left(R^{n-d}\right) \hookrightarrow L_{\vec{z}}^{q}\left(R^{n-d}\right), \frac{1}{q}=\frac{1}{r}-\frac{s_{2}}{n-d}$ (note that $s_{2}<$ $\frac{n-d}{2}$ and one can deduce $s_{2}<\frac{n-d}{r}$ ) and $H_{\vec{y}}^{s_{1}, \rho}\left(R^{d}\right) \hookrightarrow L_{\vec{y}}^{\infty}\left(R^{d}\right), \frac{1}{q}=\frac{1}{\rho}-\frac{s_{1}}{d}$ (note that $s_{1}<$ $\frac{d}{2}$ and one can deduce $s_{1}<\frac{d}{\rho}$ ). Similar to the proof of Lemma 3.2, the inequality can be established. 


\section{Proof of theorems}

Proof of Theorem 1.1 The solution of the initial value problem (1.1) is equivalent to the integral equation

$$
u(t)=S(t) \varphi-i \int_{0}^{t} S(t-\tau)|u|^{\alpha} u(\tau) d \tau
$$

In order to use the Banach fixed point theorem, we can define the mapping $T$ as follows:

$$
T u=S(t) \varphi-i \int_{0}^{t} S(t-\tau)|u|^{\alpha} u(\tau) d \tau
$$

(1) Take the case $s_{1}=0, s_{2}=0$. When $2 n-d>4$ and $\frac{-(2 n-d-4)+\sqrt{(2 n-d-4)^{2}+32(2 n-d)}}{2(2 n-d)}<\alpha<$ $\frac{8}{2 n-d-4}$, taking $\rho=r=\alpha+2, \theta=\frac{8-(2 n-d-4) \alpha}{4 \alpha(\alpha+2)}$. Let $X=\left\{u:(0,+\infty) \rightarrow L_{\vec{y}}^{\rho} L_{\vec{z}}^{r}\right\}$, we take the norm in $X$ to be $\|u\|_{X}=\sup _{t>0} t^{\theta}\|u(t)\|_{L_{\tilde{y}}^{\rho} L_{\tilde{z}}^{r}}$. We define the metric space $\left(X_{1}^{1}, d\right)$ as follows:

$$
\begin{aligned}
& X_{1}^{1}=\left\{u(t) \in X \mid\|u\|_{X} \leq 2 \varepsilon\right\}, \\
& d(u, v)=\sup _{t>0} t^{\theta}\|u(t)-v(t)\|_{L_{\bar{y}}^{\rho} L_{\vec{z}}^{r}}, \quad \forall u, v \in X_{1}^{1} .
\end{aligned}
$$

Obviously, we can prove that $\left(X_{1}^{1}, d\right)$ is a complete metric space.

We first prove that $T$ maps $X_{1}^{1}$ into itself. Indeed, from Lemma 2.1 and Lemma 3.1 we have

$$
\begin{aligned}
& t^{\theta}\|T u\|_{L_{\bar{y}}^{\rho} L_{\bar{z}}^{r}} \leq t^{\theta}\|S(t) \varphi\|_{L_{\tilde{y}}^{\rho} L_{\bar{z}}^{r}}+t^{\theta} \int_{0}^{t}\left\|S(t-\tau)|u|^{\alpha} u(\tau)\right\|_{L_{\vec{y}}^{\rho} L_{\bar{z}}^{r}} d \tau \\
& \leq t^{\theta}\|S(t) \varphi\|_{L_{\vec{y}}^{\rho} L_{\bar{z}}^{r}}+t^{\theta} \int_{0}^{t} c|t-\tau|^{-\frac{n-d}{2}\left(1-\frac{2}{r}\right)}|t-\tau|^{-\frac{d}{4}\left(1-\frac{2}{\rho}\right)}\left\||u|^{\alpha} u\right\|_{L_{\vec{y}}^{\rho^{\prime}} L_{\tilde{z}}^{r^{\prime}}} d \tau \\
& \leq t^{\theta}\|S(t) \varphi\|_{L_{\bar{y}}^{\rho} L_{\bar{z}}^{r}}+c t^{\theta} \int_{0}^{t}|t-\tau|^{*}\|u\|_{L_{\bar{y}}^{\rho} L_{\bar{z}}^{r}}^{\alpha+1} d \tau \\
& =t^{\theta}\|S(t) \varphi\|_{L_{\bar{y}}^{\rho} L_{\bar{z}}^{r}}+c t^{\theta} \int_{0}^{t} \tau^{-\theta(\alpha+1)}|t-\tau|^{*}\left(\tau^{\theta}\|u\|_{L_{\bar{y}}^{\rho} L_{\bar{z}}^{r}}\right)^{\alpha+1} d \tau \\
& \leq t^{\theta}\|S(t) \varphi\|_{L_{\bar{y}}^{\rho} L_{\bar{z}}^{r}}+c t^{\theta}\|u\|_{X}^{\alpha+1} \int_{0}^{t} \tau^{-\theta(\alpha+1)}|t-\tau|^{*} d \tau \\
& =t^{\theta}\|S(t) \varphi\|_{L_{\tilde{y}}^{\rho} L_{\bar{z}}^{r}}+c\|u\|_{X}^{\alpha+1} t^{\theta-\theta(\alpha+1)+*+1} \int_{0}^{1}\left(\frac{\tau}{t}\right)^{-\theta(\alpha+1)}\left|1-\frac{\tau}{t}\right|^{*} d\left(\frac{\tau}{t}\right) \\
& =t^{\theta}\|S(t) \varphi\|_{L_{\tilde{y}}^{\rho} L_{\bar{z}}^{r}}+c\|u\|_{X}^{\alpha+1} t^{\theta-\theta(\alpha+1)+*+1} B(1-\theta(\alpha+1), 1+*),
\end{aligned}
$$

where $*=-\frac{n-d}{2}\left(1-\frac{2}{r}\right)-\frac{d}{4}\left(1-\frac{2}{\rho}\right), B(\cdot, \cdot)$ is a Beta function.

Notice that $\theta-\theta(\alpha+1)+*+1=0$, which is

$$
\theta \alpha=1-\frac{n-d}{2}\left(1-\frac{2}{r}\right)-\frac{d}{4}\left(1-\frac{2}{\rho}\right),
$$

and

$$
\theta(\alpha+1)<1, \quad \frac{n-d}{2}\left(1-\frac{2}{r}\right)+\frac{d}{4}\left(1-\frac{2}{\rho}\right)<1 .
$$


It follows that

$$
t^{\theta}\|T u\|_{L_{\vec{y}}^{\rho} L_{\vec{z}}^{r}} \leq\|S(t) \varphi\|_{X}+c\|u\|_{X}^{\alpha+1}
$$

Hence

$$
\|T u\|_{X} \leq \varepsilon+c\|u\|_{X}^{\alpha+1}
$$

Now let $\varepsilon<\left(\frac{1}{c 2^{\alpha+1}}\right)^{\frac{1}{\alpha}}$, we obtain $\|T u\|_{X} \leq 2 \varepsilon$. That is to say, $T$ maps $X_{1}^{1}$ into itself. Furthermore

$$
\begin{aligned}
& t^{\theta}\|T u-T v\|_{L_{\vec{y}}^{\rho} L_{\vec{z}}^{r}} \\
& =t^{\theta}\left\|-i \int_{0}^{t} S(t-\tau)|u|^{\alpha} u(\tau) d \tau+i \int_{0}^{t} S(t-\tau)|v|^{\alpha} \nu(\tau) d \tau\right\|_{L_{\vec{y}}^{\rho} L_{\vec{z}}^{r}} \\
& \leq t^{\theta} \int_{0}^{t}|t-\tau|^{*}\left\||u|^{\alpha} u(\tau)-|v|^{\alpha} v(\tau)\right\|_{L_{\vec{y}}^{\rho^{\prime}} L_{\vec{z}}^{r^{\prime}}} d \tau \\
& \leq c t^{\theta} \int_{0}^{t}|t-\tau|^{*}\left(\|u\|_{L_{\vec{y}}^{\rho} L_{\bar{z}}^{r}}^{\alpha}+\|v\|_{L_{\vec{y}}^{\rho} L_{\vec{z}}^{r}}^{\alpha}\right)\|u(\tau)-v(\tau)\|_{L_{\vec{y}}^{\rho} L_{\vec{z}}^{r}} d \tau \\
& =c t^{\theta} \int_{0}^{t}|t-\tau|^{*} \tau^{-\theta(\alpha+1)}\left(\tau^{\theta \alpha}\|u\|_{L_{\vec{y}}^{\rho} L_{\vec{z}}^{r}}^{\alpha}+\tau^{\theta \alpha}\|v\|_{L_{\vec{y}}^{\rho} L_{\vec{z}}^{r}}^{\alpha}\right) \tau^{\theta}\|u(\tau)-v(\tau)\|_{L_{\vec{y}}^{\rho} L_{\vec{z}}^{r}} d \tau \\
& \leq c t^{\theta} \int_{0}^{t}|t-\tau|^{*} \tau^{-\theta(\alpha+1)}\left(\|u\|_{X}^{\alpha}+\|v\|_{X}^{\alpha}\right)\|u-v\|_{X} d \tau \\
& =c t^{\theta-\theta(\alpha+1)+*+1} \int_{0}^{1}\left(\frac{\tau}{t}\right)^{-\theta(\alpha+1)}\left|1-\frac{\tau}{t}\right|^{*}\left(\|u\|_{X}^{\alpha}+\|v\|_{X}^{\alpha}\right)\|u-v\|_{X} d\left(\frac{\tau}{t}\right) .
\end{aligned}
$$

Similar to the above proof, we obtain

$$
t^{\theta}\|T u-T v\|_{L_{\vec{y}}^{\rho} L_{\vec{z}}^{r}} \leq c\left(\|u\|_{X}^{\alpha}+\|v\|_{X}^{\alpha}\right)\|u-v\|_{X} \leq 2 c(2 \varepsilon)^{\alpha}\|u-v\|_{X} .
$$

Since $\varepsilon<\left(\frac{1}{c 2^{\alpha+1}}\right)^{\frac{1}{\alpha}},\|T u-T v\|_{X}<\|u-v\|_{X}$. We can also see that $T$ is a contraction mapping from $X_{1}^{1}$ into $X_{1}^{1}$.

Thus by the Banach fixed point theorem, we see that $T$ has a unique fixed point $u \in$ $X_{1}^{1} \subset X$ which is the global solution of initial value problem (1.1).

(2) Take the case $0 \leq s_{1}<\frac{d}{2}, 0<s_{2}$. We divide it into three cases:

(2.1): The subcase $0<s_{2}<(n-d) / 2$, when

$$
\begin{aligned}
& \frac{-\left(2 n-d-4 s_{2}-2 s_{1}-4\right)+\sqrt{\left(2 n-d-4 s_{2}-2 s_{1}-4\right)^{2}+32\left(2 n-d-4 s_{2}-2 s_{1}\right)}}{2\left(2 n-d-4 s_{2}-2 s_{1}\right)} \\
& \quad<\alpha<\frac{8}{2 n-d-4 s_{2}-2 s_{1}-4},
\end{aligned}
$$

taking $\theta=\frac{8-\left(2 n-d-4 s_{2}-2 s_{1}-4\right) \alpha}{4 \alpha(\alpha+2)}, \rho=\frac{d(\alpha+2)}{d+s_{1} \alpha}, r=\frac{(\alpha+2)(n-d)}{n-d+\alpha s_{2}}$. Let $X=\left\{u:(0,+\infty) \rightarrow H_{\vec{y}}^{s_{1}, \rho} H_{\vec{z}}^{s_{2}, r}\right\}$, we take the norm in $X$ to be $\|u\|_{X}=\sup _{t>0} t^{\theta}\|u(t)\|_{H_{\vec{y}}^{s_{1}, \rho}} H_{\vec{z}}^{s_{2}, r}$. We define the metric space $\left(X_{2}^{1}, d\right)$ as follows:

$$
X_{2}^{1}=\left\{u \in X \mid\|u\|_{X} \leq 2 \varepsilon\right\}
$$




$$
d(u, v)=\sup _{t>0} t^{\theta}\|u(t)-v(t)\|_{L_{\bar{y}}^{\rho} L_{\vec{z}}^{r}}, \quad \forall u, v \in X_{2}^{1} .
$$

Obviously, we can prove that $\left(X_{2}^{1}, d\right)$ is a complete metric space.

(2.2): The subcase $s_{2}=(n-d) / 2$, when $\frac{-\left(2 n-d-2 s_{1}-4\right)+\sqrt{\left(2 n-d-2 s_{1}-4\right)^{2}+32\left(2 n-d-2 s_{1}\right)}}{2\left(2 n-d-2 s_{1}\right)}<\alpha<$ $\frac{8}{2 n-d-2 s_{1}-4}$, taking $\theta=\frac{8-\left(2 n-d-2 s_{1}-4\right) \alpha}{4 \alpha(\alpha+2)}, \rho=\frac{d(\alpha+2)}{d+s_{1} \alpha}, r=\alpha+2$. Let $X=\left\{u:(0,+\infty) \rightarrow H_{\vec{y}}^{s_{1}, \rho} H_{\vec{z}}^{s_{2}, r}\right\}$,

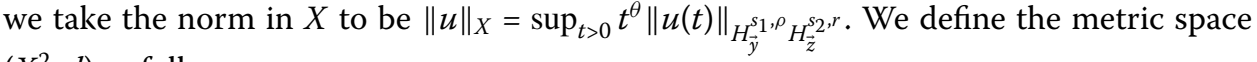
$\left(X_{2}^{2}, d\right)$ as follows:

$$
\begin{aligned}
& X_{2}^{2}=\left\{u \in X \mid\|u\|_{X} \leq 2 \varepsilon\right\}, \\
& d(u, v)=\sup _{t>0} t^{\theta}\|u(t)-v(t)\|_{H_{\bar{y}}^{s_{1}, \rho} L_{\bar{z}}^{r}}, \quad \forall u, v \in X_{2}^{2} .
\end{aligned}
$$

Obviously, we can prove that $\left(X_{2}^{2}, d\right)$ is a complete metric space.

(2.3): The subcase $s_{2}>(n-d) / 2$, when $\frac{-\left(d-2 s_{1}-4\right)+\sqrt{\left(d-2 s_{1}-4\right)^{2}+32\left(d-2 s_{1}\right)}}{2\left(d-2 s_{1}\right)}<\alpha<\frac{8}{d-2 s_{1}-4}$, taking $\theta=\frac{8-\left(d-2 s_{1}-4\right) \alpha}{4 \alpha(\alpha+2)}, \rho=\frac{d(\alpha+2)}{d+s_{1} \alpha}, r=2$. Let $X=\left\{u:(0,+\infty) \rightarrow H_{\vec{y}}^{s_{1}, \rho} H_{\vec{z}}^{s_{2}, r}\right\}$, we take the norm in $X$ to be $\|u\|_{X}=\sup _{t>0} t^{\theta}\|u(t)\|_{H_{1}^{s_{1}, \rho}, H_{z}^{s_{2}, r}}$. We define the metric space $\left(X_{2}^{3}, d\right)$ as follows:

$$
\begin{aligned}
& X_{2}^{3}=\left\{u \in X \mid\|u\|_{X} \leq 2 \varepsilon\right\}, \\
& d(u, v)=\sup _{t>0} t^{\theta}\|u(t)-v(t)\|_{L_{\bar{y}} L_{\vec{z}}^{r}}, \quad \forall u, v \in X_{2}^{3} .
\end{aligned}
$$

Obviously, we can prove that $\left(X_{2}^{3}, d\right)$ is a complete metric space.

Using the Banach fixed point theorem, by Lemma 3.7, Lemma 3.5 and Lemma 3.2 we see that $T u$ has a unique fixed point in $X_{2}^{1}, X_{2}^{2}$ and $X_{2}^{3}$ which is the global solution of initial value problem (1.1).

(3) Take the case $s_{1}=\frac{d}{2}, 0<s_{2}$. We divide it into three cases:

(3.1): The subcase $0<s_{2}<(n-d) / 2$, when $\frac{-\left(2 n-d-4 s_{2}-4\right)+\sqrt{\left(2 n-d-4 s_{2}-4\right)^{2}+32\left(2 n-d-4 s_{2}\right)}}{2\left(2 n-d-4 s_{2}\right)}<\alpha<$ $\frac{8}{2 n-d-4 s_{2}-4}$, taking $\theta=\frac{8-\left(2 n-d-4 s_{2}-4\right) \alpha}{4 \alpha(\alpha+2)}, \rho=\alpha+2, r=\frac{(\alpha+2)(n-d)}{n-d+\alpha s_{2}}$. Let $X=\{u:(0,+\infty) \rightarrow$ $\left.H_{\vec{y}}^{s_{1}, \rho} H_{\vec{z}}^{s_{2}, r}\right\}$, we take the norm in $X$ to be $\|u\|_{X}=\sup _{t>0} t^{\theta}\|u(t)\|_{H_{1}^{s_{1}, \rho} \rho} H_{\bar{z}}^{s_{2}, r}$. We define the metric space $\left(X_{3}^{1}, d\right)$ as follows:

$$
\begin{aligned}
& X_{3}^{1}=\left\{u \in X \mid\|u\|_{X} \leq 2 \varepsilon\right\}, \\
& d(u, v)=\sup _{t>0} t^{\theta}\|u(t)-v(t)\|_{L_{\bar{y}}^{\rho} H_{\bar{z}}^{\delta_{2}, r},}, \quad \forall u, v \in X_{3}^{1} .
\end{aligned}
$$

Obviously, we can prove that $\left(X_{3}^{1}, d\right)$ is a complete metric space.

(3.2): The subcase $s_{2}=(n-d) / 2$, when $\frac{-(2 n-d-4)+\sqrt{(2 n-d-4)^{2}+32(2 n-d)}}{2(2 n-d)}<\alpha<\frac{8}{2 n-d-4}$, taking $\theta=\frac{8-(2 n-d-4) \alpha}{4 \alpha(\alpha+2)}, \rho=\alpha+2, r=\alpha+2$. Let $X=\left\{u:(0,+\infty) \rightarrow H_{\vec{y}}^{s_{1}, \rho} H_{\vec{z}}^{s_{2}, r}\right\}$, we take the norm in $X$ to be $\|u\|_{X}=\sup _{t>0} t^{\theta}\|u(t)\|_{H_{y}^{s_{1}}, \rho} H_{z}^{s_{2}}, r$. We define the metric space $\left(X_{3}^{2}, d\right)$ as follows:

$$
\begin{aligned}
& X_{3}^{2}=\left\{u \in X \mid\|u\|_{X} \leq 2 \varepsilon\right\}, \\
& d(u, v)=\sup _{t>0} t^{\theta}\|u(t)-v(t)\|_{L_{\tilde{y}}^{\rho} L_{\bar{z}}^{r}}, \quad \forall u, v \in X_{3}^{2} .
\end{aligned}
$$

Obviously, we can prove that $\left(X_{3}^{2}, d\right)$ is a complete metric space. 
(3.3): The subcase $s_{2}>(n-d) / 2$, when $\frac{-(d-4)+\sqrt{(d-4)^{2}+32 d}}{2 d}<\alpha<\frac{8}{d-4}$, taking $\theta=\frac{8-(d-4) \alpha}{4 \alpha(\alpha+2)}$, $\rho=\alpha+2, r=2$. Let $X=\left\{u:(0,+\infty) \rightarrow H_{\vec{y}}^{s_{1}, \rho} H_{\vec{z}}^{s_{2}, r}\right\}$, we take the norm in $X$ to be $\|u\|_{X}=$

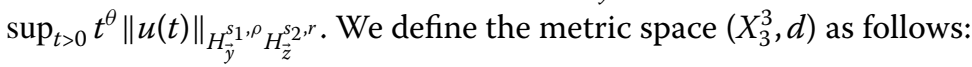

$$
\begin{aligned}
& X_{3}^{3}=\left\{u \in X \mid\|u\|_{X} \leq 2 \varepsilon\right\}, \\
& d(u, v)=\sup _{t>0} t^{\theta}\|u(t)-v(t)\|_{L_{\vec{y}}^{\rho} H_{\bar{z}}^{s_{2}, r}, \quad \forall u, v \in X_{3}^{3} .}
\end{aligned}
$$

Obviously, we can prove that $\left(X_{3}^{3}, d\right)$ is a complete metric space.

Using Lemma 3.4, Lemma 3.6 and the Banach fixed point theorem again, we obtain the global solution of the problem (1.1).

(4) Take the case $s_{1}>\frac{d}{2}, 0 \leq s_{2}$. We divide it into two cases:

(4.1): The subcase $0 \leq s_{2}<(n-d) / 2$, when $\frac{-\left(n-d-2 s_{2}-2\right)+\sqrt{\left(n-d-2 s_{2}-2\right)^{2}+16\left(n-d-2 s_{2}\right)}}{2\left(n-d-2 s_{2}\right)}<\alpha<$ $\frac{4}{n-d-2 s_{2}-2}$, taking $\theta=\frac{4-\left(n-d-2 s_{2}-2\right) \alpha}{2 \alpha(\alpha+2)}, \rho=2, r=\frac{(\alpha+2)(n-d)}{n-d+\alpha s_{2}}$, let $X=\left\{u:(0,+\infty) \rightarrow H_{\vec{y}}^{s_{1}, \rho} H_{\vec{z}}^{s_{2}, r}\right\}$, we take the norm in $X$ to be $\|u\|_{X}=\sup _{t>0} t^{\theta}\|u(t)\|_{H_{\vec{y}}^{s_{1}}, \rho} H_{\vec{z}}^{s_{2}, r}$. We define the metric space $\left(X_{4}^{1}, d\right)$ as follows:

$$
\begin{aligned}
& X_{4}^{1}=\left\{u \in X \mid\|u\|_{X} \leq 2 \varepsilon\right\}, \\
& d(u, v)=\sup _{t>0} t^{\theta}\|u(t)-v(t)\|_{L_{\vec{y}}^{\rho} L_{\vec{z}}^{r}, \quad \forall u, v \in X_{4}^{1} .}
\end{aligned}
$$

Obviously, we can prove that $\left(X_{4}^{1}, d\right)$ is a complete metric space.

(4.2): The subcase $s_{2}=(n-d) / 2$, when $\frac{-(n-d-2)+\sqrt{(n-d-2)^{2}+16(n-d)}}{2(n-d)}<\alpha<\frac{4}{n-d-2}$, taking $\theta=$ $\frac{4-(n-d-2) \alpha}{2 \alpha(\alpha+2)}, \rho=2, r=\alpha+2$, let $X=\left\{u:(0,+\infty) \rightarrow H_{\vec{y}}^{s_{1}, \rho} H_{\vec{z}}^{s_{2}, r}\right\}$, we take the norm in $X$ to be $\|u\|_{X}=\sup _{t>0} t^{\theta}\|u(t)\|_{H_{y}^{s_{1}}, \rho} H_{z}^{s_{2}, r}$. We define the metric space $\left(X_{4}^{2}, d\right)$ as follows:

$$
\begin{aligned}
& X_{4}^{2}=\left\{u \in X \mid\|u\|_{X} \leq 2 \varepsilon\right\}, \\
& d(u, v)=\sup _{t>0} t^{\theta}\|u(t)-v(t)\|_{H_{\vec{y}}^{s_{1}}, \rho} L_{\vec{z}}^{r}, \quad \forall u, v \in X_{4}^{2} .
\end{aligned}
$$

Obviously, we can prove that $\left(X_{4}^{2}, d\right)$ is a complete metric space.

Similar to the proof of (1), by Lemma 3.3 and Lemma 3.5, it is known from the Banach fixed point theorem that we have the existence of a unique fixed point of $u \in X$ which is the global solution of the initial value problem of (1.1).

Proof of Theorem 1.2 Here we only prove the decay estimate of solution in the case $s_{1}=0$, $s_{2}=0$ and the rest is similar.

We first prove the continuous dependence of the solution on the initial value. $\varphi(x)$ and $\psi(x)$ satisfy the initial condition, and $u, v$ are the two solutions of problem (1.1) corresponding to initial value $\varphi, \psi$, respectively. We know $T u=u, T v=v$ by Theorem 1.1 and from the proof of Theorem 1.1 we can obtain

$$
\begin{gathered}
t^{\theta}\|u(t)-v(t)\|_{L_{\vec{y}}^{\rho} L_{\vec{z}}^{r}} \\
=t^{\theta}\|T u-T v\|_{L_{\vec{y}}^{\rho} L_{\vec{z}}^{r}}
\end{gathered}
$$




$$
\begin{aligned}
& \leq t^{\theta}\|S(t)(\varphi-\psi)\|_{L_{\bar{y}}^{\rho} L_{\bar{z}}^{r}}+t^{\theta} \int_{0}^{t}\left\|S(t-\tau)\left(|u|^{\alpha} u(\tau)-|v|^{\alpha} \nu(\tau)\right)\right\|_{L_{\tilde{y}}^{\rho} L_{\bar{z}}^{r}} d \tau \\
& \leq t^{\theta}\|S(t)(\varphi-\psi)\|_{L_{\bar{y}}^{\rho} L_{\bar{z}}^{r}}+2 c(2 \varepsilon)^{\alpha}\|u-v\|_{X} .
\end{aligned}
$$

Since $2 c(2 \varepsilon)^{\alpha}<1$,

$$
\|u-v\|_{X} \leq\|S(t)(\varphi-\psi)\|_{X}
$$

In the following we prove the decay estimate of the solution

$$
\begin{aligned}
& t^{\theta}(1+t)^{\eta} \int_{0}^{t}\left\|S(t-\tau)\left(|u|^{\alpha} u(\tau)-|v|^{\alpha} v(\tau)\right)\right\|_{L_{\bar{y}}^{\rho} L_{\bar{z}}^{r}} d \tau \\
& \leq t^{\theta}(1+t)^{\eta} \int_{0}^{t}|t-\tau|^{*}\left\||u|^{\alpha} u(\tau)-|v|^{\alpha} \nu(\tau)\right\|_{L_{\vec{y}}^{\rho^{\prime}} L_{\vec{z}}^{r^{\prime}}} d \tau \\
& \leq c t^{\theta}(1+t)^{\eta} \int_{0}^{t}|t-\tau|^{*}\left(\|u\|_{L_{\bar{y}}^{\rho} L_{\bar{z}}^{r}}^{\alpha}+\|v\|_{L_{\bar{y}}^{\rho} L_{\bar{z}}^{r}}^{\alpha}\|u(\tau)-v(\tau)\|_{L_{\bar{y}}^{\rho} L_{\bar{z}}^{r}} d \tau\right. \\
& \leq c t^{\theta}(1+t)^{\eta} \int_{0}^{t} \tau^{-\theta(\alpha+1)}(1+\tau)^{-\eta}|t-\tau|^{*}\left(\tau^{\theta \alpha}\|u\|_{L_{\tilde{y}}^{\rho} L_{\bar{z}}^{r}}^{\alpha}+\tau^{\theta \alpha}\|v\|_{L_{\tilde{y}}^{\rho} L_{\bar{z}}^{r}}^{\alpha}\right) \\
& \cdot \tau^{\theta}(1+\tau)^{\eta}\|u(\tau)-v(\tau)\|_{L_{\bar{y}}^{\rho} L_{\bar{z}}^{r}} d \tau \\
& \leq c t^{\theta}(1+t)^{\eta} \int_{0}^{t} \tau^{-\theta(\alpha+1)}(1+\tau)^{-\eta}|t-\tau|^{*}\left(\sup _{0<\tau<t} \tau^{\theta \alpha}\|u\|_{L_{\bar{y}}^{\rho} L_{\bar{z}}^{r}}^{\alpha}+\sup _{0<\tau<t} \tau^{\theta \alpha}\|v\|_{L_{L_{\bar{y}}}^{\rho} L_{\bar{z}}^{r}}^{\alpha}\right) \\
& \cdot \sup _{0<\tau<t} \tau^{\theta}(1+\tau)^{\eta}\|u(\tau)-v(\tau)\|_{L_{\bar{y}}^{\rho} L_{\bar{z}}^{r}} d \tau \\
& \leq 2 c(2 \varepsilon)^{\alpha} t^{\theta} \sup _{0<\tau<t} \tau^{\theta}(1+\tau)^{\eta}\|u(\tau)-v(\tau)\|_{L_{\tilde{y}}^{\rho} L_{\bar{z}}^{r}} \int_{0}^{t}\left(\frac{1+t}{1+\tau}\right)^{\eta}|t-\tau|^{*}|\tau|^{-\theta(\alpha+1)} d \tau \\
& \leq 2 c(2 \varepsilon)^{\alpha} t^{\theta} \sup _{t>0} t^{\theta}(1+t)^{\eta}\|u(t)-v(t)\|_{L_{L_{\bar{y}}}^{\rho} L_{\bar{z}}^{r}} \int_{0}^{t}\left(\frac{t}{\tau}\right)^{\eta}|t-\tau|^{*}|\tau|^{-\theta(\alpha+1)} d \tau \\
& =2 c(2 \varepsilon)^{\alpha} t^{\theta+\eta} \sup _{t>0} t^{\theta}(1+t)^{\eta}\|u(t)-v(t)\|_{L_{\bar{y}}^{\rho} L_{\bar{z}}^{r}} \int_{0}^{t}|t-\tau|^{*}|\tau|^{-\theta(\alpha+1)-\eta} d \tau
\end{aligned}
$$

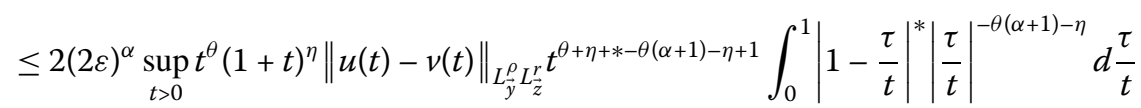

$$
\begin{aligned}
& =2 c(2 \varepsilon)^{\alpha} \sup _{t>0} t^{\theta}(1+t)^{\eta}\|u(t)-v(t)\|_{L_{\tilde{y}}^{\rho} L_{\bar{z}}^{r}} t^{\theta+*-\theta(\alpha+1)+1} B(1-\theta(\alpha+1)-\eta, 1+*),
\end{aligned}
$$

where $*=-\frac{n-d}{2}\left(1-\frac{2}{r}\right)-\frac{d}{4}\left(1-\frac{2}{\rho}\right), B(\cdot, \cdot)$ is a Beta function.

Notice that $\theta-\theta(\alpha+1)+*+1=0$, and $\theta(\alpha+1)+\eta<1, \frac{n-d}{2}\left(1-\frac{2}{r}\right)+\frac{d}{4}\left(1-\frac{2}{\rho}\right)<1$, thus we have

$$
\begin{aligned}
& t^{\theta}(1+t)^{\eta} \int_{0}^{t}\left\|S(t-\tau)\left(|u|^{\alpha} u(\tau)-|v|^{\alpha} v(\tau)\right)\right\|_{L_{\tilde{y}}^{\rho} L_{\bar{z}}^{r}} d \tau \\
& \leq 2 c(2 \varepsilon)^{\alpha} \sup _{t>0} t^{\theta}(1+t)^{\eta}\|u(t)-v(t)\|_{L_{\bar{y}}^{\rho} L_{\bar{z}}^{r},}
\end{aligned}
$$


so that

$$
\begin{aligned}
& \sup _{t>0} t^{\theta}(1+t)^{\eta}\|u(t)-v(t)\|_{L_{\tilde{y}}^{\rho} L_{\vec{z}}^{r}} \\
& \leq \sup _{t>0} t^{\theta}(1+t)^{\eta}\|S(t)(\varphi-\psi)\|_{L_{\vec{y}}^{\rho} L_{\vec{z}}^{r}} \\
& \quad+\sup _{t>0} t^{\theta}(1+t)^{\eta} \int_{0}^{t}\left\|S(t-\tau)\left(|u|^{\alpha} u(\tau)-|v|^{\alpha} v(\tau)\right)\right\|_{L_{\bar{y}}^{\rho} L_{\vec{z}}^{r}} d \tau \\
& \leq \sup _{t>0} t^{\theta}(1+t)^{\eta}\|S(t)(\varphi-\psi)\|_{L_{\vec{y}}^{\rho} L_{\vec{z}}^{r}}+2 c(2 \varepsilon)^{\alpha} \sup _{t>0} t^{\theta}(1+t)^{\eta}\|u(t)-v(t)\|_{L_{\vec{y}}^{\rho} L_{\vec{z}}^{r} \cdot}
\end{aligned}
$$

Since $2 c(2 \varepsilon)^{\alpha}<1$ and $\sup _{t>0} t^{\theta}(1+t)^{\eta}\|S(t)(\varphi-\psi)\|_{L_{\vec{y}}^{\rho} L_{\vec{z}}^{r}}<+\infty$ we have

$$
\sup _{t>0} t^{\theta}(1+t)^{\eta}\|u(t)-v(t)\|_{L_{\vec{y}}^{\rho} L_{\vec{z}}^{r}} \leq c .
$$

Hence

$$
\|u(t)-v(t)\|_{L_{\vec{y}}^{\rho} L_{\vec{z}}^{r}} \leq c t^{-\theta}(1+t)^{-\eta} .
$$

\section{Acknowledgements}

The authors would like to thank the anonymous referee for her/his useful comments and valuable suggestions.

\section{Funding}

This work is supported by Natural Science Foundation of China (No. 11571209, No. 61473180, No. 61503230).

\section{Competing interests}

The authors declare that they have no competing interests.

\section{Authors' contributions}

The authors declare that the study was realized in collaboration with the same responsibility. All authors read and approved the final manuscript.

\section{Publisher's Note}

Springer Nature remains neutral with regard to jurisdictional claims in published maps and institutional affiliations.

Received: 18 December 2018 Accepted: 24 April 2019 Published online: 08 May 2019

\section{References}

1. Aceves, A.B., De Angelis, C., Turitsyn, S.K.: Multidimensional solitons in fiber arrays. Opt. Lett. 19, 329-331 (1995)

2. Wen, S., Fan, D.: Spatiotemporal instabilities in nonlinear Kerr media in the presence of arbitrary higher order dispersions. J. Opt. Soc. Am. B 19, 1653-1659 (2002)

3. Fibich, G., Ilan, B., Schochet, S.: Critical exponents and collapse of nonlinear Schrödinger equations with anisotropic fourth-order dispersion. Nonlinearity 16, 1809-1821 (2003)

4. Fibich, G., Papanicolaou, G.C.: A modulation method for self-focusing in the perturbed critical nonlinear Schrödinger equation. Phys. Lett. A 239, 167-173 (1998)

5. Fibich, G., Papanicolaou, G.C.: Self-focusing in the perturbed and unperturbed nonlinear Schrödinger equation in critical dimension. SIAM J. Appl. Math. 60, 183-240 (1999)

6. Dinh, V.D.: On well-posedness, regularity and ill-posedness for the nonlinear fourth-order Schrödinger equation. Bull. Belg. Math. Soc. Simon Stevin 25, 415-437 (2018)

7. Hao, C., Hsiao, L., Wang, B.: Well-posedness for the fourth-order Schrödinger equations. J. Math. Anal. Appl. 320 246-265 (2006)

8. Hao, C., Hsiao, L., Wang, B.: Well-posedness of the Cauchy problem for the fourth-order Schrödinger equations in high dimensions. J. Math. Anal. Appl. 328, 58-83 (2007)

9. Pausader, B.: Global well-posedness for energy critical fourth-order Schrödinger equations in the radial case. Dyn. Partial Differ. Equ. 4, 197-225 (2007)

10. Pausader, B.: The cubic fourth-order Schrödinger equation. J. Funct. Anal. 256, 2473-2517 (2009)

11. Segata, J.: Well-posedness for the fourth-order nonlinear Schrödinger type equation related to the vortex filament. Differ. Integral Equ. 16, 841-864 (2003)

12. Ozsari, T., Yolcu, N.: The initial-boundary value problem for the biharmonic Schrödinger equation on the half-line. Commun. Pure Appl. Anal. (2019). https://arxiv.org/abs/1802.10499 
13. Capistrano-Filho, R.A., Cavalcante, M., Gallego, F.A.: Lower regularity solutions of the biharmonic Schrödinger equation in a quarter plane (2018). https://arxiv.org/abs/1812.11079

14. Guo, C.H., Cui, S.B.: Well-posedness of the Cauchy problem of high dimension non-isotropic fourth-order Schrödinger equations in Sobolev spaces. Nonlinear Anal. 70, 3761-3772 (2009)

15. Zhao, X., Cui, S.: Global small solutions of the Cauchy problem for non-isotropic Schrödinger equations. Kyungpook Math. J. 48, 101-108 (2008)

16. Bouchel, O.: Remarks on NLS with higher order anisotropic dispersion. Adv. Differ. Equ. 13, 169-198 (2008)

17. Guo, C., Cui, S.: Solvability of the Cauchy problem of non-isotropic Schrödinger equations in Sobolev spaces. Nonlinear Anal. 68, 768-780 (2008)

18. Saut, J.C., Segata, J.: Asymptotic behavior in time of solution to the nonlinear Schrödinger equation with higher order anisotropic dispersion. Discrete Contin. Dyn. Syst. 39, 219-239 (2019)

19. Zhao, X.Q., Guo, C.H., Sheng, W.C., Wei, X.M.: Well-posedness of the fourth-order perturbed Schrödinger type equation in non-isotropic Sobolev spaces. J. Math. Anal. Appl. 382, 97-109 (2011)

20. Guo, C., Zhao, X., Wei, X.: Cauchy problem for higher-order Schrödinger equations in anisotropic Sobolev space. Appl. Anal. 88, 1329-1338 (2009)

21. Ben-Artzi, M., Koch, H., Saut, J.-C.: Dispersion estimates for fourth order Schrödinger equations. C. R. Acad. Sci. Paris, Sér. I Math. 330, 87-92 (2000)

22. Cui, S.B., Guo, C.H.: Well-posedness of higher-order nonlinear Schrödinger equations in Sobolev spaces $H^{5}\left(R^{n}\right)$ and applications. Nonlinear Anal. 67, 687-707 (2006)

23. Christ, F.M., Weinstein, M.I.: Dispersive of small-amplitude solutions of the generalized Korteweg-de Vries equation J. Funct. Anal. 100, 87-109 (1991)

24. Runst, T., Sickel, W.: Sobolev Spaces of Fractional Order, Nemytskii Operators and Nonlinear Partial Differential Equations. de Gruyter, Berlin (1996)

\section{Submit your manuscript to a SpringerOpen ${ }^{\circ}$ journal and benefit from:}

- Convenient online submission

- Rigorous peer review

- Open access: articles freely available online

- High visibility within the field

- Retaining the copyright to your article

Submit your next manuscript at $\gg$ springeropen.com 